\title{
Characterization of vB_ApiM_fHyAci03, a novel lytic bacteriophage that infects clinical Acinetobacter strains
}

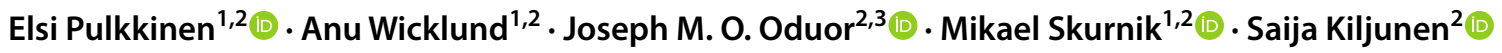

Received: 27 November 2018 / Accepted: 17 April 2019 / Published online: 23 May 2019

(c) The Author(s) 2019

\begin{abstract}
We present here the isolation and characterization of Acinetobacter pittii phage vB_ApiM_fHyAci03 (fHyAci03), which belongs to the family Myoviridae. The fHyAci03 genome was found to be 165,975 bp in length and predicted to contain 255 genes. While the whole genome was $92.4 \%$ identical to Acinetobacter baumannii phage KARL-1, phylogenetic analysis based on phage long distal tail fiber amino acid sequences assigned fHyAci03 and KARL-1 to different subclusters, reflecting their different host species. Together with phylogenetic analysis, genome comparisons indicated that fHyAci03 is a novel member of the subfamily Tevenvirinae. Host range experiments revealed that fHyAci03 could infect two clinical strains of Acinetobacter nosocomialis and six clinical strains of A. pittii. Thus, fHyAci03 is a novel lytic phage that infects clinical Acinetobacter strains and represents a potential new candidate to be used in phage therapy.
\end{abstract}

The genus Acinetobacter includes multiple nosocomial opportunistic pathogens. Within this genus, A. baumannii, A. pittii, and A. nosocomialis are the most frequently isolated species from hospitalized patients around the world [1]. Recently, these bacteria have become a public health concern because of the growing tendency to develop antibiotic resistance [2]. Due to the continued increase in multidrugresistant (MDR) bacterial strains, there has been a great deal of recent interest in phage therapy research.

fHyAci03 was isolated from a municipal sewage sample collected in Hyvinkää, Finland, using clinical A. pittii strain \#5565 (obtained from HUSLAB, Helsinki, Finland) as the host. The morphology of fHyAci03 was examined by

Handling Editor: Johannes Wittmann.

Electronic supplementary material The online version of this article (https://doi.org/10.1007/s00705-019-04284-z) contains supplementary material, which is available to authorized users.

Saija Kiljunen

saija.kiljunen@helsinki.fi

1 Division of Clinical Microbiology, HUSLAB, University of Helsinki and Helsinki University Hospital, Helsinki, Finland

2 Human Microbiome Research Program, Faculty of Medicine, University of Helsinki, Helsinki, Finland

3 Department of Medical Microbiology, KAVI-ICR, University of Nairobi, Nairobi, Kenya transmission electron microscopy as described previously [3] (Supplementary Fig. 1). The dimensions of the prolate head were $111 \pm 5.2 \mathrm{~nm}$ (length) and $82 \pm 5.1 \mathrm{~nm}$ (width), and the tail length was $90 \pm 4 \mathrm{~nm}$. Dimensions were calculated based on ten virions. Together with the genomic information, the morphological characteristics indicated that fHyAci03 belongs to the family Myoviridae and the subfamily Tevenvirinae. fHyAci03 host range was tested with 48 clinical Acinetobacter strains (Supplementary Table 1). Host range experiments revealed that $\mathrm{fHyAci03}$ could infect two out of three A. nosocomialis strains, and six out of $18 \mathrm{~A}$. pittii strains that were tested. Phage DNA was isolated using an Invisorb Spin Virus DNA Minikit (Stratec Biomedical). Next-generation sequencing was performed at the Institute for Molecular Medicine Finland (FIMM), using a DNA library that was constructed using a Nextera Sample Prep Kit (Illumina, San Diego, CA, USA). Paired-end sequencing was done using an Illumina MiSeq sequencer (Illumina, San Diego, CA, USA) with a read length of 300 nucleotides. Draft sequences were assembled using two pipelines in parallel, Geneious software, version 10.1, and the A5-miseq integrated pipeline [4]. The average whole-genome read coverage was 42.9x. Results from both pipelines were compared to identify a single consensus sequence. To manually verify the fidelity of the assembly, the reads were mapped back to the contigs using Geneious (mean coverage 43.0x, range, 11-96x). The genome was found to be $165,975 \mathrm{bp}$ in length, with a mean GC content of $36.8 \%$. The genome was 


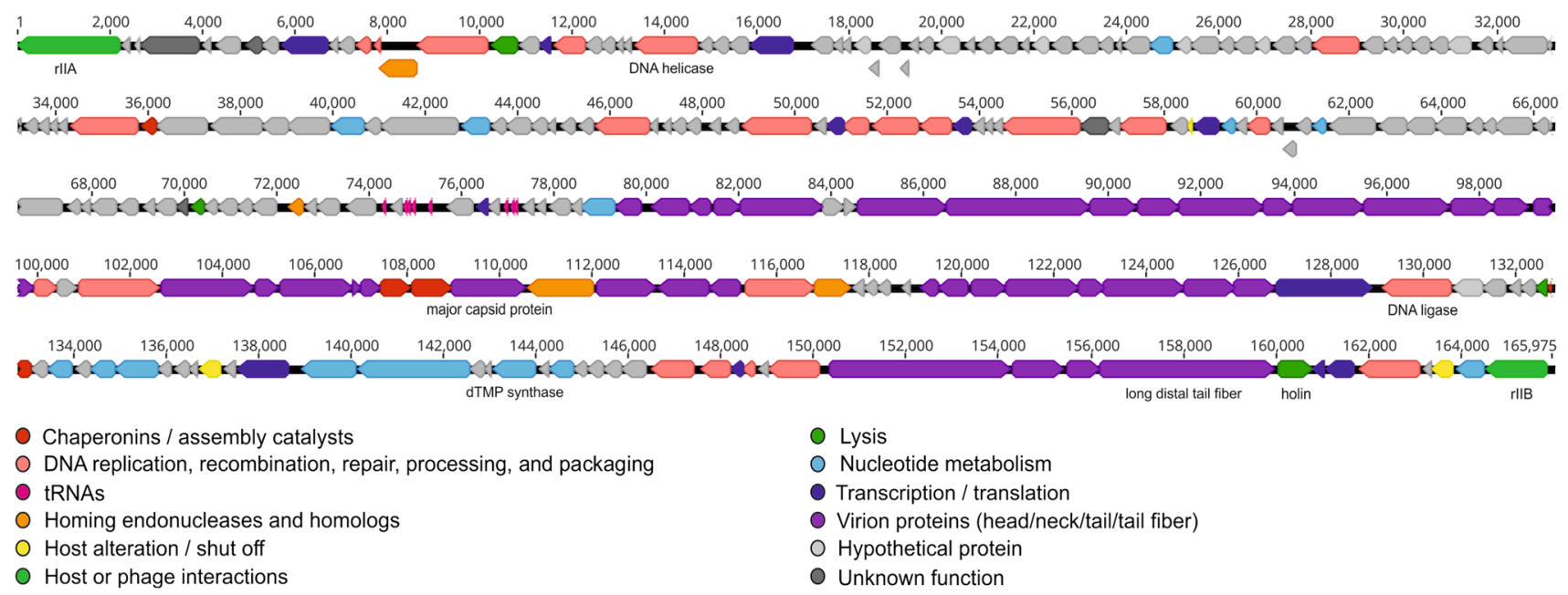

Fig. 1 Annotated genome map of fHyAci03. The predicted 247 protein-coding and eight tRNA genes are colour coded according to the key. Some examples of genes that are conserved within the subfamily Tevenvirinae are indicated

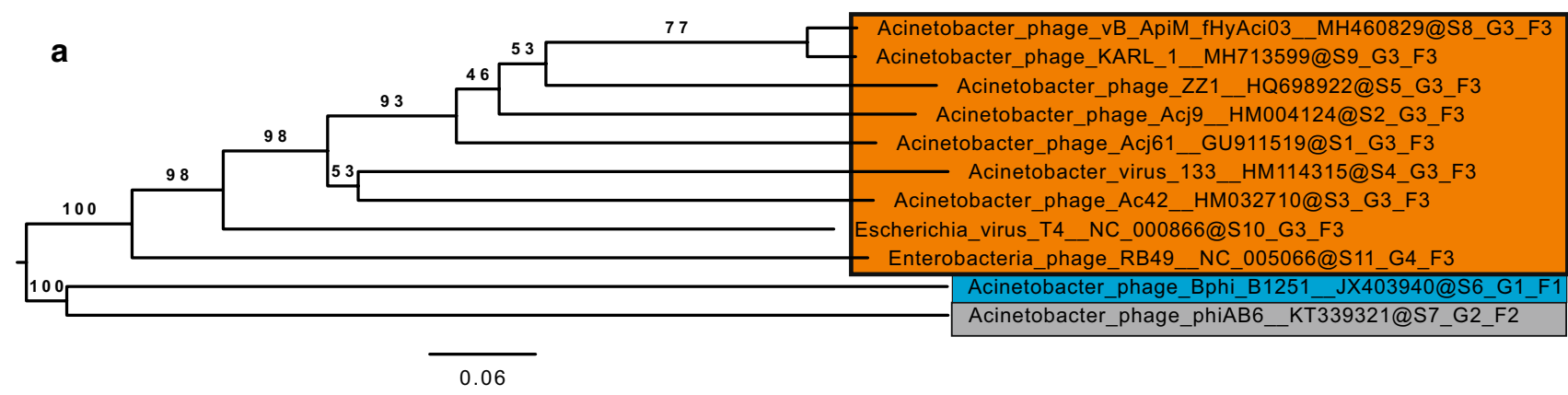

b

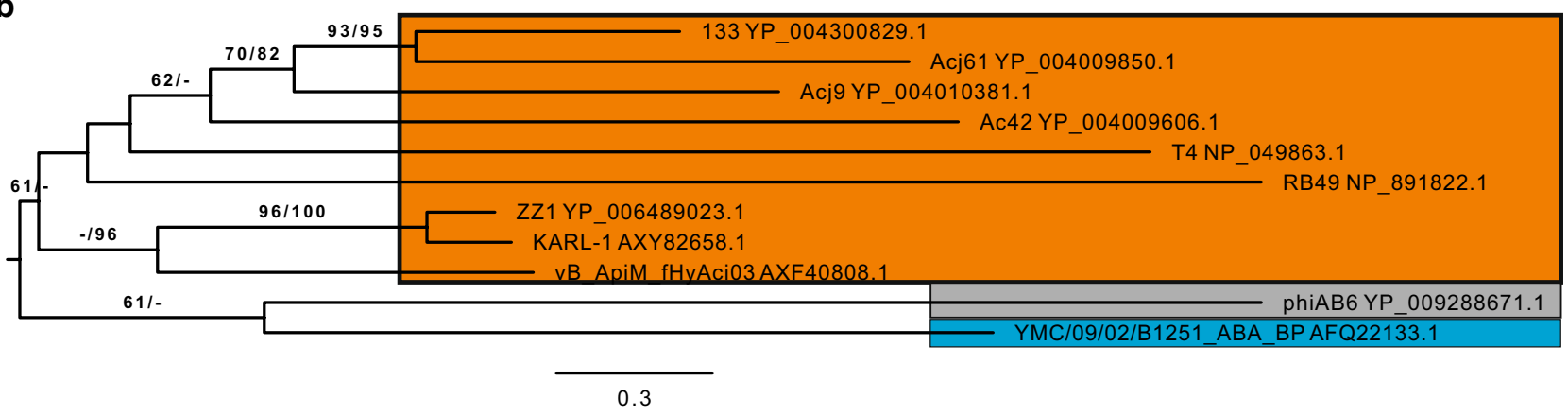

Fig. 2 Phylogenetic trees based on the whole-genome nucleotide sequences (a) and amino acid sequences of the tail fiber proteins (b) showing the relationships within the subfamily Tevenvirinae and

annotated using Geneious software, RAST [5], BLASTP [6], ARAGORN [7], tRNAscan-SE version 2.0 [8], ResFinder-3.1 [9], and VirulenceFinder-2.0 [10]. fHyAci03 contained 255 predicted genes, eight of which code for tRNAs (Supplementary table S2) and 247 for proteins (Fig. 1). No toxin-, virulence-factor-, antibiotic-resistance-, or lysogeny-related genes were found, indicating that $\mathrm{fHyAci03}$ is between the families Myoviridae, Podoviridae, and Siphoviridae. Phages are color coded according to family/subfamily. Orange, Myoviridae, Tevenvirinae; blue, Siphoviridae; grey, Podoviridae

strictly lytic and a potential new candidate for phage therapy. A BLASTn search revealed that the most closely related phages belonged to the subfamily Tevenvirinae (Supplementary Table S3). Alignment with EMBOSS Stretcher showed that fHyAci03 is $92.4 \%$ identical to A. baumannii phage KARL-1 (MH713599.1) [11]. Prior to the sequence comparisons, the sequence of KARL-1 was rearranged with 
respect to its orientation and starting point to align maximally with the T-even phages. Genome-wide nucleotide phylogenetic analysis using VICTOR (Virus Classification and Tree Building Online Resource) [12] placed fHyAci03 in the same subcluster with KARL-1 (Fig. 2A). Further phylogenetic analysis based on phage tail fiber amino acid sequences using VICTOR assigned fHyAci03 and KARL-1 to different subclusters (Fig. 2B), reflecting the difference in host species. Comparisons of conserved gene product content between fHyAci03, KARL1, and other members of the subfamily Tevenvirinae, were performed using CoreGenes3.5 [13] (Supplementary Table S4). Based on shared gene content $(96.76 \%$ shared homologs) and nucleotide sequence similarity, we suggest that fHyAci03 and KARL-1 could comprise a new genus within the subfamily Tevenvirinae, for which we propose the name "FHyAci03virus" after the first sequenced isolate. To conclude, fHyAci03 is a novel lytic phage that, with further characterization, could represent an interesting new candidate for phage therapy.

\section{Nucleotide sequence accession number}

The genomic sequence of fHyAci03 has been deposited in the GenBank database under the accession number MH460829.1.

Acknowledgements Open access funding provided by University of Helsinki including Helsinki University Central Hospital.

Funding The Jane \& Aatos Erkko Foundation and the Helsinki University Hospital Special State Subsidy for Health Science Research (project TYH2018228) are acknowledged for funding.

\section{Compliance with ethical standards}

Conflict of interest The authors declare no conflict of interest.

Open Access This article is distributed under the terms of the Creative Commons Attribution 4.0 International License (http://creativeco mmons.org/licenses/by/4.0/), which permits unrestricted use, distribution, and reproduction in any medium, provided you give appropriate credit to the original author(s) and the source, provide a link to the Creative Commons license, and indicate if changes were made.

\section{References}

1. Lee K, Yong D, Jeong SH, Chong Y (2011) Multidrug-resistant Acinetobacter spp.: increasingly problematic nosocomial pathogens. Yonsei Med J 52(6):879-891. https://doi.org/10.3349/ ymj.2011.52.6.879
2. Manchanda V, Sanchaita S, Singh NP (2010) Multidrug resistant Acinetobacter. J Glob Infect Dis 2(3):291-304. https://doi. org/10.4103/0974-777X.68538

3. Leskinen K, Tuomala H, Wicklund A, Horsma-Heikkinen J, Kuusela P, Skurnik M, Kiljunen S (2017) Characterization of vB_SauM-fRuSau02, a Twort-like bacteriophage isolated from a therapeutic phage cocktail. Viruses 9(9):E258. https://doi. org/10.3390/v9090258

4. Coil D, Jospin G, Darling AE (2015) A5-miseq: an updated pipeline to assemble microbial genomes from Illumina MiSeq data. Bioinformatics (Oxford, England) 31(4):587-589. https://doi. org/10.1093/bioinformatics/btu661

5. Brettin T, Davis JJ, Disz T, Edwards RA, Gerdes S, Olsen GJ, Olson R, Overbeek R, Parrello B, Pusch GD, Shukla M, Thomason Iii JA, Stevens R, Vonstein V, Wattam AR, Xia F (2015) RASTtk: a modular and extensible implementation of the RAST algorithm for building custom annotation pipelines and annotating batches of genomes. Sci Rep 5:8365. https://doi.org/10.1038/srep0 8365

6. Johnson M, Zaretskaya I, Raytselis Y, Merezhuk Y, McGinnis S, Madden TL (2008) NCBI BLAST: a better web interface. Nucleic Acids Res 36(Web Server issue):W5-W9. https://doi.org/10.1093/ nar/gkn201

7. Laslett D, Canback B (2004) ARAGORN, a program to detect tRNA genes and tmRNA genes in nucleotide sequences. Nucleic Acids Res 32(1):11-16. https://doi.org/10.1093/nar/gkh152

8. Lowe TM, Chan PP (2016) tRNAscan-SE On-line: integrating search and context for analysis of transfer RNA genes. Nucleic Acids Res 44(W1):W54-W57. https://doi.org/10.1093/nar/gkw41 3

9. Zankari E, Hasman H, Cosentino S, Vestergaard M, Rasmussen S, Lund O, Aarestrup FM, Larsen MV (2012) Identification of acquired antimicrobial resistance genes. J Antimicrob Chemother 67(11):2640-2644. https://doi.org/10.1093/jac/dks261

10. Joensen KG, Scheutz F, Lund O, Hasman H, Kaas RS, Nielsen EM, Aarestrup FM (2014) Real-time whole-genome sequencing for routine typing, surveillance, and outbreak detection of verotoxigenic Escherichia coli. J Clin Microbiol 52(5):1501-1510. https://doi.org/10.1128/jcm.03617-13

11. Li W, Cowley A, Uludag M, Gur T, McWilliam H, Squizzato S, Park YM, Buso N, Lopez R (2015) The EMBL-EBI bioinformatics web and programmatic tools framework. Nucleic Acids Res 43(W1):W580-W584. https://doi.org/10.1093/nar/gkv279

12. Meier-Kolthoff JP, Goker M (2017) VICTOR: genome-based phylogeny and classification of prokaryotic viruses. Bioinformatics (Oxford, England) 33(21):3396-3404. https://doi.org/10.1093/ bioinformatics/btx 440

13. Turner D, Reynolds D, Seto D, Mahadevan P (2013) CoreGenes3.5: a webserver for the determination of core genes from sets of viral and small bacterial genomes. BMC Res Notes 6:140. https://doi.org/10.1186/1756-0500-6-140

Publisher's Note Springer Nature remains neutral with regard to jurisdictional claims in published maps and institutional affiliations. 